PROCEEDINGS OF THE

AMERICAN MATHEMATICAL SOCIETY

Volume 137, Number 4, April 2009, Pages 1479-1487

S 0002-9939(08)09687-1

Article electronically published on November 3, 2008

\title{
A LOWER BOUND FOR THE CHUNG-DIACONIS-GRAHAM RANDOM PROCESS
}

\author{
MARTIN HILDEBRAND
}

(Communicated by Richard C. Bradley)

\begin{abstract}
Chung, Diaconis, and Graham considered random processes of the form $X_{n+1}=a_{n} X_{n}+b_{n}(\bmod p)$ where $p$ is odd, $X_{0}=0, a_{n}=2$ always, and $b_{n}$ are i.i.d. for $n=0,1,2, \ldots$ In this paper, we show that if $P\left(b_{n}=-1\right)=P\left(b_{n}=0\right)=P\left(b_{n}=1\right)=1 / 3$, then there exists a constant $c>1$ such that $c \log _{2} p$ steps are not enough to make $X_{n}$ get close to being uniformly distributed on the integers $\bmod p$.
\end{abstract}

\section{INTRODUCTION}

In 2], Chung, Diaconis, and Graham considered random processes of the form

$$
X_{n+1}=2 X_{n}+b_{n} \quad(\bmod p)
$$

where $X_{0}=0, b_{n}$ are i.i.d. for $n=0,1,2, \ldots$, and $p$ is odd. They focussed on the case where $P\left(b_{n}=1\right)=P\left(b_{n}=0\right)=P\left(b_{n}=-1\right)=1 / 3$. These random processes have some similarity to certain pseudorandom sequences used by computers. Subsequently some generalizations of this random process have been considered. See, for example, [1, 4], 5], and [6. Suppose $P_{n}(s)=P\left(X_{n}=s\right)$ where $s \in \mathbb{Z} / p \mathbb{Z}$. Define the variation distance of a probability $P$ on $\mathbb{Z} / p \mathbb{Z}$ from the uniform distribution $U$ on $\mathbb{Z} / p \mathbb{Z}$ by

$$
\|P-U\|=\frac{1}{2} \sum_{s \in \mathbb{Z} / p \mathbb{Z}}|P(s)-1 / p|=\max _{A \subseteq \mathbb{Z} / p \mathbb{Z}}|P(A)-U(A)| .
$$

Chung, Diaconis, and Graham showed that for almost all odd $p$, if $N \geq \frac{\log p}{\log (9 / 5)}+c$, then $\left\|P_{N}-U\right\|=O\left((5 / 9)^{c}\right)$. They also state that more complicated arguments give the following result: For any $\epsilon>0$ and almost all odd $p$, if $N \geq(\hat{c}+\epsilon) \log _{2} p$, then $\left\|P_{N}-U\right\|<\epsilon$ where

$$
\hat{c}=\left(1-\log _{2}\left(\frac{5+\sqrt{17}}{9}\right)\right)^{-1}=1.01999186 \ldots
$$

Note that the values of $X_{N}$, when viewed as integers, range from $-2^{N}+1$ to $2^{N}-1$, inclusive. Thus, if $N<(1-\epsilon) \log _{2} p$ where $\epsilon>0$ is given, the number of values in this range is at most $2 p^{1-\epsilon}-1$, and $\left\|P_{N}-U\right\|>1-\left(2 p^{1-\epsilon}-1\right) / p \rightarrow 1$ as $p \rightarrow \infty$.

Received by the editors June 2, 2008.

2000 Mathematics Subject Classification. Primary 60C05; Secondary 60B15.

(C)2008 American Mathematical Society Reverts to public domain 28 years from publication 
Chung, Diaconis, and Graham 2 speculate, "It is conceivable that in fact $(1+o(1)) \log _{2} p$ steps are enough for almost all [odd] $p$ to force $P_{N}$ to converge to uniform." However, we shall show that this statement, although described as conceivable in 2], is in fact false. In particular, we shall prove the following theorem:

Theorem 1. If $P\left(b_{n}=1\right)=P\left(b_{n}=0\right)=P\left(b_{n}=-1\right)=1 / 3$ and $X_{n}$ and $P_{n}$ are as above, then there exists a value $c_{1}>1$ such that if $n=n(p)<c_{1} \log _{2} p$, then $\left\|P_{n}-U\right\| \rightarrow 1$ as $p \rightarrow \infty$.

To motivate somewhat the proof of this theorem, we shall also prove the following theorem:

Theorem 2. If $P\left(b_{n}=1\right)=0.4, P\left(b_{n}=0\right)=0.6$, and $X_{n}$ and $P_{n}$ are as above, then there exists a value $c_{2}>1$ such that if $n=n(p)<c_{2} \log _{2} p$, then $\left\|P_{n}-U\right\| \rightarrow 1$ as $p \rightarrow \infty$.

\section{Proof of Theorem 2}

First observe that the following proposition holds:

Proposition 1. If $X_{0}=0$ and $X_{n+1}=2 X_{n}+b_{n}$ for $n \geq 0$, then

$$
X_{n}=\sum_{i=0}^{n-1} 2^{n-1-i} b_{i} .
$$

Now suppose $P\left(b_{n}=1\right)=0.4$ and $P\left(b_{n}=0\right)=0.6$. Let $A_{n}=\mid\{m: 0 \leq m \leq$ $\left.n-1, b_{m}=1\right\} \mid$. By elementary arguments, for any $\epsilon>0, P\left((0.4-\epsilon) n<A_{n}<\right.$ $(0.4+\epsilon) n) \rightarrow 1$ as $n \rightarrow \infty$. Thus, except on a set which has probability approaching 0 as $n \rightarrow \infty, X_{n}$ takes on at most

$$
\sum_{j=\lceil(0.4-\epsilon) n\rceil}^{\lfloor(0.4+\epsilon) n\rfloor}\left(\begin{array}{l}
n \\
j
\end{array}\right)
$$

different values. We shall assume that $0.4+\epsilon<0.5$. Note that Stirling's formula implies

$$
\begin{aligned}
& \sum_{j=\lceil(0.4-\epsilon) n\rceil}^{\lfloor(0.4+\epsilon) n\rfloor}\left(\begin{array}{l}
n \\
j
\end{array}\right) \\
\leq & (2 \epsilon n+1)\left(\begin{array}{c}
n \\
\lfloor(0.4+\epsilon) n\rfloor
\end{array}\right) \\
\leq & \frac{(2 \epsilon n+1) c_{3} n^{n} \sqrt{2 \pi n}}{((0.4+\epsilon) n)^{(0.4+\epsilon) n}((0.6-\epsilon) n)^{(0.6-\epsilon) n} 2 \pi \sqrt{((0.4+\epsilon) n)((0.6-\epsilon) n)}} \\
\leq & \frac{c_{3}(2 \epsilon n+1) \sqrt{2 \pi n}}{2 \pi \sqrt{((0.4+\epsilon) n)((0.6-\epsilon) n)}} \cdot \frac{1}{(0.4+\epsilon)^{(0.4+\epsilon) n}(0.6-\epsilon)^{(0.6-\epsilon) n}}
\end{aligned}
$$

where $c_{3}$ is a positive constant. Note that

$$
(0.4+\epsilon)^{(0.4+\epsilon) n}(0.6-\epsilon)^{(0.6-\epsilon) n}=2^{n\left((0.4+\epsilon) \log _{2}(0.4+\epsilon)+(0.6-\epsilon) \log _{2}(0.6-\epsilon)\right)} .
$$

It can be shown that if $0<\epsilon<0.1$, then

$$
-\left((0.4+\epsilon) \log _{2}(0.4+\epsilon)+(0.6-\epsilon) \log _{2}(0.6-\epsilon)\right)<1 .
$$


If

$$
c_{2}<\frac{1}{-\left((0.4+\epsilon) \log _{2}(0.4+\epsilon)+(0.6-\epsilon) \log _{2}(0.6-\epsilon)\right)}
$$

and $n=n(p)<c_{2} \log _{2} p$, then

$$
\frac{\sum_{j=\lceil(0.4-\epsilon) n\rceil}^{\lfloor(0.4+\epsilon) n\rfloor}\left(\begin{array}{l}
n \\
j
\end{array}\right)}{p} \rightarrow 0
$$

as $p \rightarrow \infty$. Thus $\left\|P_{n}-U\right\| \rightarrow 1$ as $p \rightarrow \infty$ if $n=n(p)<c_{2} \log _{2} p$. Note that $c_{2}$ can be chosen so that $c_{2}>1$, since

$$
\frac{1}{-\left((0.4+\epsilon) \log _{2}(0.4+\epsilon)+(0.6-\epsilon) \log _{2}(0.6-\epsilon)\right)}>1 .
$$

\section{Overview of the Proof of Theorem 1}

By Proposition 1 $X_{n}$ is determined by the $n$-tuple $\left(b_{0}, b_{1}, \ldots, b_{n-1}\right)$. However, if $P\left(b_{n}=1\right)=P\left(b_{n}=0\right)=P\left(b_{n}=-1\right)$, then many possible $n$-tuples $\left(b_{0}, b_{1}, \ldots, b_{n-1}\right)$ may give the same value for $X_{n}$. For example, if $n=3$, the 3 -tuples $(1,-1,-1),(0,1,-1)$, and $(0,0,1)$ all give $X_{3}=1$. We shall place the $n$-tuple in a standard form $\left(\tilde{b}_{0}, \tilde{b}_{1}, \ldots, \tilde{b}_{n-1}\right)$ so that

$$
2^{n-1} \tilde{b}_{0}+2^{n-2} \tilde{b}_{1}+\cdots+\tilde{b}_{n-1}=2^{n-1} b_{0}+2^{n-2} b_{1}+\cdots+b_{n-1} .
$$

In this standard form, either none of $\tilde{b}_{0}, \tilde{b}_{1}, \ldots, \tilde{b}_{n-1}$ are -1 or none of $\tilde{b}_{0}, \tilde{b}_{1}, \ldots$, $\tilde{b}_{n-1}$ are 1 . In the first case (excluding the event where $b_{0}, b_{1}, \ldots, b_{n-1}$ are all 0 ), we shall show that for every $\epsilon>0$, the number of values $a$ in $\{1, \ldots, n-1\}$ such that both $\tilde{b}_{a-1}$ and $\tilde{b}_{a}$ are 1 lies between $(4 / 18-\epsilon) n$ and $(4 / 18+\epsilon) n$, except for events which have probability approaching 0 as $n \rightarrow \infty$. Likewise, in the second case (excluding the event where $b_{0}, b_{1}, \ldots, b_{n-1}$ are all 0 ), the number of values $a$ in $\{1,2, \ldots, n-1\}$ such that both $\tilde{b}_{a-1}$ and $\tilde{b}_{a}$ are -1 lies between $((4 / 18)-\epsilon) n$ and $((4 / 18)+\epsilon) n$, except for events which have probability approaching 0 as $n \rightarrow \infty$. A Stirling's formula argument will give the theorem.

We shall divide the $n$-tuples $\left(b_{0}, b_{1}, \ldots, b_{n-1}\right)$ into three cases. In the first case, there exists a value $j$ in $\{0,1, \ldots, n-1\}$ such that $b_{j}=1$ and $b_{k}=0$ if $0 \leq k<j$. We call this case "first 1 ". In the second case, there exists a value $j$ in $\{0,1, \ldots, n-1\}$ such that $b_{j}=-1$ and $b_{k}=0$ if $0 \leq k<j$. We call this case "first -1 ". In the third case, $b_{0}=b_{1}=\cdots=b_{n-1}=0$. As $n \rightarrow \infty$, the probability of "first 1 " approaches $1 / 2$, and the probability of "first -1 " approaches $1 / 2$. (Both probabilities are $(1 / 2)\left(1-(1 / 3)^{n}\right)$.) In "first 1 ", none of $\tilde{b}_{0}, \tilde{b}_{1}, \ldots, \tilde{b}_{n-1}$ are -1 . In "first -1 ", none of $\tilde{b}_{0}, \tilde{b}_{1}, \ldots, \tilde{b}_{n-1}$ are 1 . We shall give detailed arguments for "first 1"; the arguments for "first -1 " are similar.

If we are in "first 1 ", consider the infinite sequence $\left(b_{0}, b_{1}, b_{2}, \ldots\right)$. After some leading zeroes, with probability 1 this sequence consists of strings of "blocks" $B_{1}, B_{2}, \ldots$ where each $B_{i}$ is a finite string starting with 1 and having no other 1 's in it. For example, if $\left(b_{0}, b_{1}, \ldots, b_{10}\right)=(0,0,1,-1,0,1,0,1,-1,1,1)$, then there are two leading zeroes, followed by $B_{1}=(1,-1,0), B_{2}=(1,0), B_{3}=(1,-1)$, and $B_{4}=(1)$. Also we say that the first coordinate of $B_{1}$ is $b_{2}$ and that $B_{1}$ has $b_{2}, b_{3}$, and $b_{4}$ as its coordinates. Note that the blocks $B_{1}, B_{2}, \ldots$ are i.i.d. given that we are in "first 1". 
Given an infinite series $\left(b_{0}, b_{1}, b_{2}, \ldots\right)$, technically the values $\tilde{b}_{0}, \tilde{b}_{1}, \ldots, \tilde{b}_{n-1}$ may depend on $n$. For example, if $\left(b_{0}, b_{1}, b_{2}\right)=(0,0,1)$, then $\left(\tilde{b}_{0}, \tilde{b}_{1}, \tilde{b}_{2}\right)=(0,0,1)$ and $\tilde{b}_{2}=1$ if $n=3$. If $\left(b_{0}, b_{1}, \ldots, b_{10}\right)=(0,0,1,-1,0,1,0,1,-1,1,1)$, then $\left(\tilde{b}_{0}, \tilde{b}_{1}, \ldots, \tilde{b}_{10}\right)=(0,0,0,1,0,1,0,0,1,1,1)$ and $\tilde{b}_{2}=0$ if $n=11$ even though $b_{0}, b_{1}$, and $b_{2}$ are unchanged. Suppose $a$ is such that $B_{i}$ has $b_{a}$ as one of its coordinates. Then for all $n>a$ such that $B_{i}$ does not also have $b_{n}$ as one of its coordinates, $\left(\tilde{b}_{0}, \tilde{b}_{1}, \ldots, \tilde{b}_{a}\right)$ will no longer vary with $n$.

\section{Number OF $a$ SUCH THAT $\tilde{b}_{a-1}=\tilde{b}_{a}=1$}

We shall consider several distinct ways to get values $a$ such that $\tilde{b}_{a-1}=\tilde{b}_{a}=1$. These ways are detailed in the following lemmas.

Lemma 1. Let $n_{1}$ be the number of a in $\{1, \ldots, n-1\}$ such that $\tilde{b}_{a-1}=1, \tilde{b}_{a}=1$, $b_{a-1}=1$, and $b_{a}=1$. Let $\epsilon>0$ be given. Given that we are in "first 1 ", the probability that $((1 / 18)-\epsilon) n<n_{1}<((1 / 18)+\epsilon) n$ approaches 1 as $n \rightarrow \infty$.

Lemma 2. Let $n_{2}$ be the number of a in $\{1, \ldots, n-1\}$ such that $\tilde{b}_{a-1}=1, \tilde{b}_{a}=1$, $b_{a-1}=1$, and $b_{a} \neq 1$. Then $n_{2}=0$.

Lemma 3. Let $n_{3}$ be the number of a in $\{1, \ldots, n-1\}$ such that $\tilde{b}_{a-1}=1, \tilde{b}_{a}=1$, $b_{a-1} \neq 1$, and $b_{a}=1$. Let $\epsilon>0$ be given. Given that we are in "first 1 ", the probability that $((1 / 18)-\epsilon) n<n_{3}<((1 / 18)+\epsilon) n$ approaches 1 as $n \rightarrow \infty$.

Lemma 4. Let $n_{4}$ be the number of a in $\{1, \ldots, n-1\}$ such that $\tilde{b}_{a-1}=1, \tilde{b}_{a}=1$, $b_{a-1} \neq 1$, and $b_{a} \neq 1$. Let $\epsilon>0$ be given. Given that we are in "first 1 ", the probability that $((1 / 9)-\epsilon) n<n_{4}<((1 / 9)+\epsilon) n$ approaches 1 as $n \rightarrow \infty$.

Proof of Lemma 2. If we are not in "first 1 ", then $\tilde{b}_{a}$ is never 1 . If we are in "first 1 ", then one obtains $\left(\tilde{b}_{0}, \tilde{b}_{1}, \ldots, \tilde{b}_{n-1}\right)$ from $\left(b_{0}, b_{1}, \ldots, b_{n-1}\right)$ as follows. If $j$ is such that $b_{j}=1$ and $b_{k}=0$ whenever $0 \leq k<j$, then $\tilde{b}_{k}=0$ whenever $0 \leq k<j$. Otherwise, for each $j_{0}$ such that $b_{j_{0}}=1$, let $j_{1}=\min \left(n, \min \left\{\ell: \ell>j_{0}, b_{\ell}=1\right\}\right)$. (By convention, assume that the minimum of an empty set is $\infty$.) If $b_{k}=0$ for all $k$ with $j_{0}<k<j_{1}$, then $\tilde{b}_{j_{0}}=1$ and $\tilde{b}_{k}=0$ for all $k$ with $j_{0}<k<j_{1}$. Otherwise $\tilde{b}_{j_{0}}=0$, and one can figure out the unique values for $\tilde{b}_{k}$ in $\{0,1\}$ when $j_{0}<k<j_{1}$. Lemma 2 follows.

Proof of Lemma 4. To prove Lemma 4, let $j$ be such that $b_{j}=1$ and $b_{k}=0$ whenever $0 \leq k<j$. Suppose that $a-1>j$ and $a<n$. Then $P\left(b_{a-1} \neq 1, b_{a} \neq 1\right)=$ 4/9. Suppose $j_{0}<a<j_{1}$ with $b_{j_{0}}=1$ and $j_{1}=\min \left(n, \min \left\{\ell: \ell>j_{0}, b_{\ell}=1\right\}\right)$. Given $j_{0}$ and $j_{1}$, there are $2^{j_{1}-j_{0}-1}$ possibilities for $\left(b_{j_{0}+1}, \ldots, b_{j_{1}-1}\right)$ and $2^{j_{1}-j_{0}-1}$ possibilities for $\left(\tilde{b}_{j_{0}+1}, \ldots, \tilde{b}_{j_{1}-1}\right)$. The possibilities for $\left(b_{j_{0}+1}, \ldots, b_{j_{1}-1}\right)$, which range from $(0, \ldots, 0)$ to $(-1, \ldots,-1)$, are in one-to-one correspondence with the possibilities for $\left(\tilde{b}_{j_{0}+1}, \ldots, \tilde{b}_{j_{1}-1}\right)$, which range from $(0, \ldots, 0)$ to $(1, \ldots, 1)$. Thus $P\left(\tilde{b}_{a-1}=1, \tilde{b}_{a}=1 \mid b_{a-1} \neq 1, b_{a} \neq 1\right)=1 / 4$, and $P\left(\tilde{b}_{a-1}=1, \tilde{b}_{a}=1, b_{a-1} \neq 1, b_{a} \neq\right.$ $1)=1 / 9$. Let $C_{a}=\left\{\tilde{b}_{a-1}=1, \tilde{b}_{a}=1, b_{a-1} \neq 1, b_{a} \neq 1\right\}$. Conditioned on $j$ such that $b_{j}=1$ and $b_{k}=0$ for $0 \leq k<j$, the events $C_{a}$ for $a-1>j, a<n$, and $a$ even are independent, and the events $C_{a}$ for $a-1>j, a<n$, and $a$ odd are independent. Since $P\left(j>\epsilon_{1} n\right) \rightarrow 0$ as $n \rightarrow \infty$ (given that we are in "first 1 ") for each $\epsilon_{1}>0$, Lemma 4 follows by elementary arguments. 
Proof of Lemma 1. To prove this lemma, suppose that we are in "first 1" and $b_{a-1}=$ 1 with $a$ in $\{1, \ldots, n-1\}$. Then $\tilde{b}_{a-1}=1$ and $\tilde{b}_{a}=1$ if and only if $b_{a}=1$ and $b_{k}=0$ for all $k$ with $a<k<j_{1}$ where $j_{1}=\min \left(n, \min \left\{\ell>a: b_{\ell}=1\right\}\right)$. Let us consider the infinite sequence $\left(b_{0}, b_{1}, \ldots\right)$. For positive integers $i$, let $D_{i}$ be the event that $B_{i}=(1)$ and $B_{i+1}$ has no -1 's in it. Note that $P\left(D_{i}\right)=(1 / 3)\left(\sum_{i=1}^{\infty}(1 / 3)^{i}\right)=1 / 6$. Observe that $D_{1}, D_{3}, D_{5}$, etc. are independent and that $D_{2}, D_{4}, D_{6}$, etc. are independent. Furthermore, given $\epsilon_{1}>0$, with probability approaching 1 as $n \rightarrow \infty$, the number of $a$ in $\{1, \ldots, n-1\}$ such that $b_{a-1}=1$ lies between $\left((1 / 3)-\epsilon_{1}\right) n$ and $\left((1 / 3)+\epsilon_{1}\right) n$ given that we are in "first 1". Suppose we are given $\epsilon^{\prime}>0$. Choose $\epsilon_{1}>0$ so that $\epsilon_{1}<6 \epsilon^{\prime}$. Then with probability approaching 1 as $n \rightarrow \infty$, at least $\left((1 / 18)-\epsilon^{\prime}\right) n$ events $D_{i}$ occur with $i<\left((1 / 3)-\epsilon_{1}\right) n$, while at most $\left((1 / 18)+\epsilon^{\prime}\right) n$ events $D_{i}$ occur with $i \leq\left((1 / 3)+\epsilon_{1}\right) n$. Thus, given $\epsilon^{\prime}>0$, the number of $i$ such that $D_{i}$ occurs and the first coordinate of $B_{i+1}$ is $b_{\ell}$ for some $\ell<n$ is, with probability approaching 1 as $n \rightarrow \infty$, between $\left((1 / 18)-\epsilon^{\prime}\right) n$ and $\left((1 / 18)+\epsilon^{\prime}\right) n$. This number of $i$ is within 1 of the number of $a$ in Lemma 1 the only possible difference occurs when the block $B_{i+1}$ has $b_{n}$ as one of its coordinates.

Proof of Lemma 3. To prove this lemma, suppose we are in "first 1 ", $b_{a-1} \neq 1$, and $b_{a}=1$ with $a$ in $\{1, \ldots, n-1\}$. Then $\tilde{b}_{a-1}=1$ and $\tilde{b}_{a}=1$ if and only if $b_{a-1}=-1$ and $b_{k}=0$ for all $k$ with $a<k<j_{1}$ where $j_{1}=\min \left(n, \min \left\{\ell>a: b_{\ell}=1\right\}\right)$. Let us consider the infinite sequence $\left(b_{0}, b_{1}, \ldots\right)$. For positive integers $i$, let $E_{i}$ be the event that $B_{i+1}$ has no -1 's in it and that $B_{i}$ ends with -1 . Note that $P\left(E_{i}\right)=1 / 6$, that $E_{1}, E_{3}, E_{5}, \ldots$ are independent, and that $E_{2}, E_{4}, E_{6}, \ldots$ are independent. Furthermore, given $\epsilon_{1}>0$, with probability approaching 1 as $n \rightarrow \infty$, the number of $a$ in $\{1, \ldots, n-1\}$ with $b_{a}=1$ lies between $\left((1 / 3)-\epsilon_{1}\right) n$ and $\left((1 / 3)+\epsilon_{1}\right) n$ given that we are in "first 1". Thus, given $\epsilon^{\prime}>0$, the number of $i$ such that $E_{i}$ occurs and the first coordinate of $B_{i+1}$ is $b_{\ell}$ for some $\ell<n$ is, with probability approaching 1 as $n \rightarrow \infty$, between $\left((1 / 18)-\epsilon^{\prime}\right) n$ and $\left((1 / 18)+\epsilon^{\prime}\right) n$. This number of $i$ is within 1 of the number of $a$ in Lemma the only possible difference occurs when $B_{i+1}$ has $b_{n}$ as one of its coordinates.

In conclusion, the number of $a$ in $\{1, \ldots, n-1\}$ such that $\tilde{b}_{a-1}=1$ and $\tilde{b}_{a}=1$ (given that we are in "first 1") lies, with probability approaching 1 as $n \rightarrow \infty$, between $((4 / 18)-\epsilon) n$ and $((4 / 18)+\epsilon) n$ for each $\epsilon>0$.

\section{STIRLING'S FORMULA ARGUMENT}

If the number of $a$ in $\{1, \ldots, n-1\}$ with $\tilde{b}_{a-1}=1$ and $\tilde{b}_{a}=1$ is no more than $((4 / 18)+\epsilon) n$, then either the number of odd $a$ in $\{1, \ldots, n-1\}$ with $\tilde{b}_{a-1}=1$ and $\tilde{b}_{a}=1$ is no more than $((2 / 18)+\epsilon / 2) n$ or the number of even $a$ in $\{1, \ldots, n-1\}$ with $\tilde{b}_{a-1}=1$ and $\tilde{b}_{a}$ is no more than $((2 / 18)+\epsilon / 2) n$.

Let us suppose that $n$ is even and the number of odd $a$ in $\{1, \ldots, n-1\}$ with $\tilde{b}_{a-1}=1$ and $\tilde{b}_{a}=1$ is no more than $((2 / 18)+\epsilon / 2) n$, where $\epsilon>0$ is such that $(2 / 18)+\epsilon / 2<1 / 8$. Then, the number of possible values of $\sum_{i=0}^{n-1} 2^{n-1-i} b_{i}$ if we have "first 1 " is at most

$$
\sum_{\left(\ell_{1}, \ell_{2}, \ell_{3}, \ell_{4}\right) \in R_{n}} \frac{((1 / 2) n) !}{\ell_{1} ! \ell_{2} ! \ell_{3} ! \ell_{4} !}
$$

where $R_{n}=\left\{\left(\ell_{1}, \ell_{2}, \ell_{3}, \ell_{4}\right): \ell_{1}+\ell_{2}+\ell_{3}+\ell_{4}=(1 / 2) n, \ell_{1} \leq((2 / 18)+\epsilon / 2) n\right\}$. The values $\ell_{1}, \ell_{2}, \ell_{3}$, and $\ell_{4}$ represent the number of odd $a$ in $\{1, \ldots, n-1\}$ such that 
$\tilde{b}_{a-1}=1$ and $\tilde{b}_{a}=1, \tilde{b}_{a-1}=1$ and $\tilde{b}_{a}=0, \tilde{b}_{a-1}=0$ and $\tilde{b}_{a}=1$, and $\tilde{b}_{a-1}=0$ and $\tilde{b}_{a}=0$, respectively.

For some polynomial $p_{1}(n)$ of $n$,

$$
\begin{aligned}
& \sum_{\left(\ell_{1}, \ell_{2}, \ell_{3}, \ell_{4}\right) \in R_{n}} \frac{((1 / 2) n) !}{\ell_{1} ! \ell_{2} ! \ell_{3} ! \ell_{4} !} \\
\leq & \left(\left(\frac{2}{18}+\frac{\epsilon}{2}\right) n+1\right) n^{2} \frac{\left(\frac{1}{2} n\right) !}{\left\lfloor\left(\frac{2}{18}+\frac{\epsilon}{2}\right) n\right\rfloor !\left(\left\lfloor\left(\frac{7}{54}-\frac{\epsilon}{6}\right) n\right\rfloor !\right)^{3}} \\
\leq & p_{1}(n) \frac{\left(\frac{1}{2} n\right)^{(1 / 2) n}}{\left(\left(\frac{2}{18}+\frac{\epsilon}{2}\right) n\right)^{((2 / 18)+\epsilon / 2) n}\left(\left(\frac{7}{54}-\frac{\epsilon}{6}\right) n\right)^{((7 / 54)-\epsilon / 6) 3 n}} \\
= & p_{1}(n) 2^{\left(0.5 \log _{2}(0.5)-((2 / 18)+\epsilon / 2) \log _{2}((2 / 18)+\epsilon / 2)-((7 / 18)-\epsilon / 2) \log _{2}((7 / 54)-\epsilon / 6)\right) n} .
\end{aligned}
$$

But if

$$
c_{1}<\frac{1}{0.5 \log _{2}(0.5)-\frac{2}{18} \log _{2}\left(\frac{2}{18}\right)-\frac{7}{18} \log _{2}\left(\frac{7}{54}\right)},
$$

where $c_{1}$ is constant and $n=n(p)<c_{1} \log _{2} p$, then we can choose $\epsilon>0$ so that

$$
\frac{p_{1}(n) 2^{\left(0.5 \log _{2}(0.5)-((2 / 18)+\epsilon / 2) \log _{2}((2 / 18)+\epsilon / 2)-((7 / 18)-\epsilon / 2) \log _{2}((7 / 54)-\epsilon / 6)\right) n}}{p} \rightarrow 0
$$

as $p \rightarrow \infty$.

Thus for such values $n$, at most $o(p)$ values of $\sum_{i=0}^{n-1} 2^{n-1-i} b_{i}$ occur in "first 1 " if $n$ is even and the number of odd $a$ in $\{1, \ldots, n-1\}$ with $\tilde{b}_{a-1}=1$ and $\tilde{b}_{a}=1$ is no more than $((2 / 18)+\epsilon / 2) n$. Minor adaptations of this argument apply if $n$ is odd, if we consider the number of even $a$ instead of the number of odd $a$, or if we consider "first -1 " instead of "first 1". (For example, if $n$ is odd but we still consider odd $a$ in "first 1", note that there are at most $o(p)$ different values of $X_{n-1}$ and 3 different values of $b_{n}$ to get that there are at most $o(p)$ different values of $X_{n}$ in this case.) There is only one value which is neither in "first 1 " nor in "first -1 ".

Observe that

$$
\frac{1}{0.5 \log _{2}(0.5)-\frac{2}{18} \log _{2}\left(\frac{2}{18}\right)-\frac{7}{18} \log _{2}\left(\frac{7}{54}\right)} \approx 1.001525 .
$$

Thus we may choose a value $c_{1}>1$ such that if $n=n(p)<c_{1} \log _{2} p$, then $X_{n}$ has, except for events with probability approaching 0 as $p \rightarrow \infty$, at most $o(p)$ values. Thus $\left\|P_{n}-U\right\| \rightarrow 1$ as $p \rightarrow \infty$.

\section{A LaRger value For $c_{1}$}

A more careful analysis of the proofs of Lemmas 1, 3, and 4 shows that for each $\epsilon>0$, the number of odd $a$ in $\{1, \ldots, n-1\}$ with $\tilde{b}_{a-1}=1$ and $\tilde{b}_{a}=1$ and the number of even $a$ in $\{1, \ldots, n-1\}$ with $\tilde{b}_{a-1}=1$ and $\tilde{b}_{a}=1$ both lie between $((2 / 18)-\epsilon) n$ and $((2 / 18)+\epsilon) n$ with probability approaching 1 as $n \rightarrow \infty$ given that we are in "first 1". Given $j$, the events $C_{a}$ are independent when $a$ is odd, $a-1>j$, and $a<n$, and also the events $C_{a}$ are independent when $a$ is even, $a-1>j$, and $a<n$; therefore the extension of Lemma 4 is straightforward. To see how to extend Lemma 1 consider the following argument. Let $i_{k}$ be the $k$-th odd value of $i$ such that $D_{i}$ occurs, and let $m_{k}$ be the value of $a$ such that $b_{a}$ is the first coordinate of the block $B_{1+i_{k}}$. Note that $m_{2}-m_{1}$, $m_{3}-m_{2}, m_{4}-m_{3}, \ldots$ are i.i.d. Let $p$ be the probability that $m_{2}-m_{1}$ is odd. 


\begin{tabular}{|l|c|c|c|c|}
\hline & $\begin{array}{c}b_{a-1}=0, \\
\tilde{b}_{a}=0\end{array}$ & $\begin{array}{c}\tilde{b}_{a-1}=0, \\
\tilde{b}_{a}=1\end{array}$ & $\begin{array}{c}\tilde{b}_{a-1}=1, \\
\tilde{b}_{a}=1\end{array}$ & $\begin{array}{c}b_{a-1}=1, \\
\tilde{b}_{a}=0\end{array}$ \\
\hline$b_{a-1}=1, b_{a}=1$ & 0 & 0 & $1 / 18$ & $1 / 18$ \\
\hline$b_{a-1} \neq 1, b_{a} \neq 1$ & $1 / 9$ & $1 / 9$ & $1 / 9$ & $1 / 9$ \\
\hline$b_{a-1}=0, b_{a}=1$ & $1 / 18$ & $1 / 18$ & 0 & 0 \\
\hline$b_{a-1}=-1, b_{a}=1$ & 0 & 0 & $1 / 18$ & $1 / 18$ \\
\hline$b_{a-1}=1, b_{a}=0$ & 0 & $1 / 18$ & 0 & $1 / 18$ \\
\hline$b_{a-1}=1, b_{a}=-1$ & $1 / 18$ & $1 / 18$ & 0 & 0 \\
\hline
\end{tabular}

TABLE 1. Cases for $\tilde{b}_{a-1}, \tilde{b}_{a}, b_{a-1}$, and $b_{a}$

If $m_{1}$ is even, then the sequence $m_{1}, m_{2}, m_{3}, \ldots$ consists of $r_{1}$ consecutive even values, then $r_{2}$ consecutive odd values, then $r_{3}$ consecutive even values, etc., where $r_{1}, r_{2}, r_{3}, \ldots$ are i.i.d. geometric random variables with parameter $p$. If $m_{1}$ is odd, then the sequence $m_{1}, m_{2}, m_{3}, \ldots$ consists of $r_{1}$ consecutive odd values, then $r_{2}$ consecutive even values, then $r_{3}$ consecutive odd values, etc., where $r_{1}, r_{2}, r_{3}, \ldots$ are i.i.d. geometric random variables with parameter $p$. Note that for each $\epsilon>0$, by Kolmogorov's maximal inequality (see p. 61 of Durrett [3], for example), $\max \left(\left|r_{1}-r_{2}\right|,\left|\left(r_{1}-r_{2}\right)+\left(r_{3}-r_{4}\right)\right|, \ldots,\left|\left(r_{1}-r_{2}\right)+\left(r_{3}-r_{4}\right)+\cdots+\left(r_{n-1}-r_{n}\right)\right|\right)<\epsilon n$ for even $n$ with probability approaching 1 as $n \rightarrow \infty$. Since for some positive constant $c, \max \left(r_{1}, r_{2}, \ldots, r_{n}\right)<c \ln (n)$ with probability approaching 1 as $n \rightarrow \infty$, this result and a similar result involving $D_{i}$ when $i$ is even imply that, for each $\epsilon>0$, the number of odd $a$ in $\{1, \ldots, n-1\}$ such that $b_{a-1}=1, b_{a}=1, \tilde{b}_{a-1}=1$, and $\tilde{b}_{a}=1$ minus the number of even $a$ in $\{1, \ldots, n-1\}$ with $b_{a-1}=1, b_{a}=1$, $\tilde{b}_{a-1}=1$, and $\tilde{b}_{a}=1$ has absolute value less than $\epsilon n$ with probability approaching 1 as $n \rightarrow \infty$ given that we are in "first 1 ". Thus, given $\epsilon>0$, the number of such odd $a$ lies between $((1 / 36)-\epsilon) n$ and $((1 / 36)+\epsilon) n$ with probability approaching 1 as $n \rightarrow \infty$ given that we are in "first 1". A similar argument applies for Lemma 3 .

With arguments resembling the proofs of Lemmas 1, 2, 3, and 4, one can show the following:

Lemma 5. Given that we are in "first 1 ", the number of a in $\{1, \ldots, n-1\}$ such that $\tilde{b}_{a-1}=1$ and $\tilde{b}_{a}=0$ lies, for each $\epsilon>0$, between $((5 / 18)-\epsilon) n$ and $((5 / 18)+\epsilon) n$ with probability approaching 1 as $n \rightarrow \infty$.

Lemma 6. Given that we are in "first 1 ", the number of a in $\{1, \ldots, n-1\}$ such that $\tilde{b}_{a-1}=0$ and $\tilde{b}_{a}=0$ lies, for each $\epsilon>0$, between $((4 / 18)-\epsilon) n$ and $((4 / 18)+\epsilon) n$ with probability approaching 1 as $n \rightarrow \infty$.

Lemma 7. Given that we are in "first 1 ", the number of a in $\{1, \ldots, n-1\}$ such that $\tilde{b}_{a-1}=0$ and $\tilde{b}_{a}=1$ lies, for each $\epsilon>0$, between $((5 / 18)-\epsilon) n$ and $((5 / 18)+\epsilon) n$ with probability approaching 1 as $n \rightarrow \infty$.

While the details are not shown here, Table 1 outlines the arguments to be shown. For example, the entry $1 / 18$ for $b_{a-1}=1, b_{a}=-1, \tilde{b}_{a-1}=0$, and $\tilde{b}_{a}=0$ means that the number of $a$ in $\{1, \ldots, n-1\}$ with $b_{a-1}=1, b_{a}=-1, \tilde{b}_{a-1}=0$, and $\tilde{b}_{a}=0$ lies, for a given $\epsilon>0$, between $((1 / 18)-\epsilon) n$ and $((1 / 18)+\epsilon) n$ with probability approaching 1 as $n \rightarrow \infty$ given that we are in "first 1 ". 
More careful arguments (similar to the extensions of Lemmas 10, 3, and 4) show that the number of odd $a$ in $\{1, \ldots, n-1\}$ with $\tilde{b}_{a-1}=1$ and $\tilde{b}_{a}=0$ lies between $((5 / 36)-\epsilon) n$ and $((5 / 36)+\epsilon) n$ (for a given $\epsilon>0)$ with probability approaching 1 as $n \rightarrow \infty$ given that we are in "first 1". Similar statements hold for even $a$ here; similar statements (where $2 / 18$ replaces $4 / 18$ and $5 / 36$ replaces $5 / 18$ ) also hold for odd $a$ and even $a$ in Lemmas 6 and 7.

The total number of possible values of $\sum_{i=0}^{n-1} 2^{n-1-i} b_{i}$ (except for events with probability approaching 0 as $n \rightarrow \infty$ ) in "first 1 " is at most (for even $n$ )

$$
\sum_{\left(\ell_{1}, \ell_{2}, \ell_{3}, \ell_{4}\right) \in S_{n}}\left(\begin{array}{c}
\frac{1}{2} n \\
\ell_{1}, \ell_{2}, \ell_{3}, \ell_{4}
\end{array}\right)
$$

with $S_{n}=\left\{\left(\ell_{1}, \ell_{2}, \ell_{3}, \ell_{4}\right): \ell_{1}+\ell_{2}+\ell_{3}+\ell_{4}=(1 / 2) n,((4 / 36)-\epsilon) n<\ell_{1}<\right.$ $((4 / 36)+\epsilon) n,((5 / 36)-\epsilon) n<\ell_{2}<((5 / 36)+\epsilon) n,((5 / 36)-\epsilon) n<\ell_{3}<((5 / 36)+$ $\left.\epsilon) n,((4 / 36)-\epsilon) n<\ell_{4}<((4 / 36)+\epsilon) n\right\}$.

A Stirling's formula argument shows that if

$$
c_{1}<\frac{1}{0.5 \log _{2}(0.5)-\frac{4}{18} \log _{2}\left(\frac{4}{36}\right)-\frac{5}{18} \log _{2}\left(\frac{5}{36}\right)}
$$

and $n=n(p)<c_{1} \log _{2} p$ where $c_{1}$ is a constant, then

$$
\sum_{\left(\ell_{1}, \ell_{2}, \ell_{3}, \ell_{4}\right) \in S_{n}}\left(\begin{array}{c}
\frac{1}{2} n \\
\ell_{1}, \ell_{2}, \ell_{3}, \ell_{4}
\end{array}\right)
$$

is $o(p)$. For odd $n$ or "first -1 ", similar arguments can be used. Thus if $n=n(p)<$ $c_{1} \log _{2} p, X_{n}$ has $o(p)$ possible different values except for events with probability approaching 0 as $p \rightarrow \infty$. Thus $\left\|P_{n}-U\right\| \rightarrow 1$ as $p \rightarrow \infty$.

Note that

$$
\frac{1}{0.5 \log _{2}(0.5)-\frac{4}{18} \log _{2}\left(\frac{4}{36}\right)-\frac{5}{18} \log _{2}\left(\frac{5}{36}\right)} \approx 1.00448 .
$$

Thus there is a gap between this lower bound and the best upper bound claimed in Chung, Diaconis, and Graham 2]. Exploring this gap is a potential problem for further study.

\section{ACKNOWLEDGMENTS}

The author thanks Ron Graham for mentioning this problem in a talk at a conference on the mathematics of Persi Diaconis in 2005. The author also thanks Persi Diaconis for encouragement.

\section{REFERENCES}

[1] Asci, C. "Generating uniform random vectors". J. Theoret. Probab. 14 (2001), 333-356. MR1838732(2002c:60115)

[2] Chung, F., Diaconis, P., and Graham, R. "Random walks arising in random number generation". Ann. Probab. 15 (1987), 1148-1165. MR893921 (88d:60033)

[3] Durrett, R. Probability: Theory and Examples, third edition. Wadsworth \& Brooks/Cole, Pacific Grove, CA, 2005. MR 1068527 (91m:60002)

[4] Hildebrand, M. "Random processes of the form $X_{n+1}=a_{n} X_{n}+b_{n}(\bmod p)$ ". Ann. Probab. 21 (1993), 710-720. MR1217562 (94d:60012) 
[5] Hildebrand, M. "Random processes of the form $X_{n+1}=a_{n} X_{n}+b_{n}(\bmod p)$ where $b_{n}$ takes on a single value". pp. 153-174, Random Discrete Structures, ed. Aldous and Pemantle. Springer-Verlag, New York, 1996. MR.1395613 (97g:60085)

[6] Hildebrand, M. "On the Chung-Diaconis-Graham random process". Electron. Commun. Probab. 11 (2006), 347-356. MR2274529 (2008g:60017)

Department of Mathematics and Statistics, University at Albany, State University of New York, Albany, New York 12222 